\title{
Dimensional Analysis of a Hot-arm Actuator
}

\author{
Thomas M. Adams \\ Rose-Hulman Institute of Technology \\ 5500 Wabash Ave, Terre Haute, IN, USA \\ adams1@rose-hulman.edu
}

\begin{abstract}
In the present work, a dimensional analysis of hot-arm actuation is performed. Several dimensionless pi groups result, including the Biot number; a thermo-electro parameter combining voltage, electrical resistivity, and thermal expansion coefficient; and a number of geometric parameters. The dimensional analysis proves that there is no way to incorporate the dimensions of Young's modulus in the problem, verifying suspicions voiced in a small number of earlier works. Furthermore, the thermo-electro parameter validates the results of many models and experiments in which deflection is directly proportional to both the thermal expansion coefficient and the square of the applied voltage. Lastly, the small Biot numbers $(0.001<B i<0.2)$ encountered in hot arm actuators indicate that a lumped-element approach to thermal modelling is legitimate, and therefore capable of producing accurate results.
\end{abstract}

Keywords: Hot arm actuators, MEMS, MEMS actuation, MEMS transducers

\section{Introduction}

Hot-arm actuators, also known as Comtois actuators or heatuators, represent one of the most common actuation methods in micro-electro-mechanical systems (MEMS) devices. Operating at lower voltages and with more favourable force-displacement characteristics than electrostatic actuation, hot-arm actuators make use of the thermal expansion of a heated member via Joule heating, resulting in mechanical stress in the device that in turn produces actuation [1]. Applications include the actuation of optical devices and the production of micromotors to name a few [2]-[3].

Error! Reference source not found. illustrates the operating principle of a hot arm actuator. Electric current flows through the hot arm, cold arm, and flexure, respectively, due to a voltage applied across the two contact pads. The high electrical resistance of the thin hot arm causes it to dissipate more thermal energy than the thicker cold arm, its temperature therefore increasing significantly. (Hence the name "hot arm.") The accompanying thermal expansion of the hot arm consequently exceeds that of the cold arm, inducing a mechanical stress that in turn causes the whole assembly to bend [4]. Typical lengths of such actuators measure $200 \mu \mathrm{m}$.

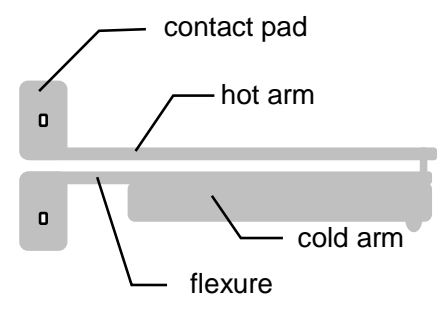

(a)

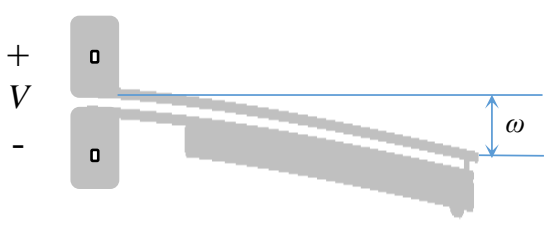

(b)

Fig. 1: A hot arm actuator: (a) Actuator in the undeflected position. (b) Actuator after a voltage is applied across the contact pads.

Physical modelling of such devices poses many difficulties, one of the most significant being that the thermal, electrical, and mechanical domains are coupled with one another. Nonetheless, many researchers have produced models of varying complexity [4-11]. In order to accommodate the large spatial variations in properties, the use of finite element analysis (FEA) software packages in such models is common [10], as are highly elaborate analytical models that solve for 
spatially distributed parameters, resulting in a large number of simultaneous equations [8]-[9]. Though these models often make accurate predictions, a lack of insight into the underlying physics can result due their inherent complexity and large computational requirements.

In [4], Adams and Layton present a simple, lumped-element model of a heat-actuator in which a single, closed form equation predicting hot-arm deflection as a function of applied voltage results. Produced as a pedagogical tool, the model incorporates many simplifications that limit its predictive accuracy. However, in [11] Adams et al expand the model, resulting in predictions that are in very good agreement with experimental results. A common feature of both these models is a lumped element approach for predicting temperatures, as is the fact that the resulting hot arm deflection has no dependence on Young's modulus. Both of these facets have otherwise gone unnoticed.

\section{Dimensionless Analysis}

The purpose of the present work is to ascertain the appropriate dimensionless groups that describe hot arm actuation. The dimensional analysis focuses on the quantities resulting from the thermal, electrical, and mechanical energy domains while deemphasizing geometric effects.

\subsection{Derivation of Dimensionless Groups}

In order to simplify the process, the dimensional analysis is performed on the simplified hot-arm actuator modelled by Adams and Layton [4]; that is, an actuator with hot arm and cold arm only, with no flexure. (Fig. 2.) The purpose is to separate the admittedly complex effect of geometry on actuation from thermal, electrical, and solid mechanics effects. The resulting dimensionless groups involving the latter quantities are nonetheless valid for all actuators. The analysis follows the Method of Repeating variables for creating dimensionless pi groups as outlined in [12].
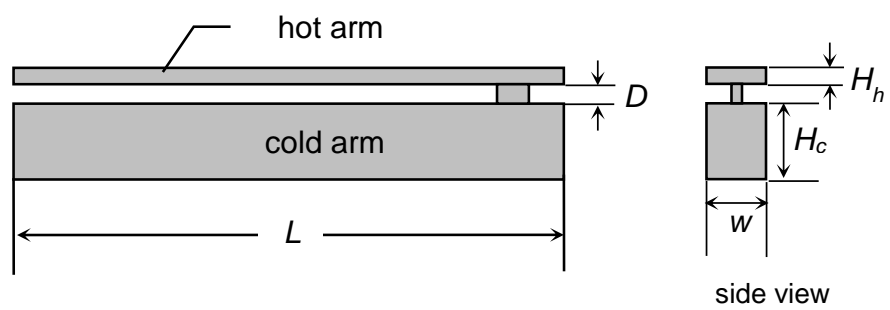

Fig. 2: Simplified hot arm actuator for which dimensional analysis is performed.

For the actuator pictured in Fig 2, the proposed functional relationship between tip deflection, $\omega$, and other parameters is given by

$$
\omega=\omega\left(L, w, H_{h}, H_{c}, D, h, E, V, \rho, \alpha, k\right)
$$

where $w$ is the thickness of the actuator (into the page), $h$ is the heat transfer coefficient between the actuator and the surroundings, $E$ is Young modulus of the actuator material, $V$ is applied voltage, $\rho$ electrical resistivity, $\alpha$ is the thermal expansion coefficient, and $k$ is the thermal conductivity of the actuator material. Taking energy (E), length (L), temperature $(\theta)$, time $(T)$, and electric charge $(\mathrm{Q})$ to be fundamental dimensions, the dimensions of the variables are

$$
\begin{aligned}
& \omega \doteq[\mathrm{L}] \quad L \doteq[\mathrm{L}] \quad w \doteq[\mathrm{L}] \quad H_{h} \doteq[\mathrm{L}] \quad H_{c} \doteq[\mathrm{L}] \quad D \doteq[\mathrm{L}] \quad h \doteq[\mathrm{E}][\mathrm{T}]^{-1}[\mathrm{~L}]^{-2}[\theta]^{-1} \\
& E \doteq[\mathrm{E}][\mathrm{L}]^{-3} \quad V \doteq[\mathrm{E}][\mathrm{Q}]^{-1} \quad \rho \doteq[\mathrm{E}][\mathrm{T}][\mathrm{L}][\mathrm{Q}]^{-2} \quad \alpha \doteq[\theta]^{-1} \quad k \doteq[\mathrm{E}][\mathrm{T}]^{-1}[\mathrm{~L}]^{-1}[\theta]^{-1} \text {. }
\end{aligned}
$$

The total number of variables in the relation given in Eq. 1 is $s=12$ requiring $r=5$ reference dimensions (length, energy, charge, time and temperature). The Buckingham Pi theorem allows the functional form of Eq. 1 to be reduced to a relationship among $n=s-r=7$ dimensionless pi groups:

$$
\Pi_{1}=\Pi_{1}\left(\Pi_{2}, \Pi_{3}, \Pi_{4}, \Pi_{5}, \Pi_{6}, \Pi_{7}\right) .
$$


Five of these pi terms are geometric in nature and are readily found by inspection:

$$
\Pi_{1}=\omega / w \quad \Pi_{2}=L / w \quad \Pi_{3}=H_{h} / w \quad \Pi_{4}=H_{c} / w \quad \Pi_{5}=D / w .
$$

Taking the repeating variables to be $w, V, \alpha, h$, and $E$, the remaining two dimensionless pi group are formed from the product of the non-repeating variables and each repeating variable raised to the appropriate power, as shown in Eq. 3:

$$
\Pi=X w^{a} V^{b} \alpha^{c} h^{d} E^{e},
$$

where $X$ is a generic non-repeating variable. In the case of thermal conductivity of the actuator material, Eq. 3 becomes

$$
\Pi_{6}=k w^{a} V^{b} \alpha^{c} h^{d} E^{e},
$$

the dimensions of which are given by

$$
\Pi_{6} \doteq[\mathrm{E}][\mathrm{T}]^{-1}[\mathrm{~L}]^{-1}[\theta]^{-1}[\mathrm{~L}]^{a}[\mathrm{~L}]^{b}[\mathrm{~T}]^{-b}[\theta]^{-c}[\mathrm{E}]^{d}[\mathrm{~T}]^{-d}[\mathrm{~L}]^{-2 d}[\theta]^{-d}[\mathrm{E}]^{e}[\mathrm{~L}]^{-3 e},
$$

or

$$
\Pi_{6} \doteq[\mathrm{E}]^{1+b+c+d}[\mathrm{~T}]^{-1-b}[\mathrm{~L}]^{-1+a-2 b-3 c}[\theta]^{-1-b-e} .
$$

For $\Pi_{6}$ to be dimensionless, $a=-1, b=-1$, and $c=d=e=0$, giving

$$
\Pi_{6}=k / h w,
$$

the inverse of which is the more familiar Biot number,

$$
B i=h w / k
$$

The remaining pi term is formed by the same method, this time using actuator resistivity as the non-repeating variable:

$$
\Pi_{7}=\rho w^{a} V^{b} \alpha^{c} h^{d} E^{e}
$$

The required exponents are $a=1, b=-2, c=-1, d=-1$, and $e=0$ :

$$
\Pi_{7}=\frac{\rho w h}{\alpha V^{2}}
$$

Taking the inverse, we arrive at what can be termed a thermo-electro parameter:

$$
P o=\frac{\alpha V^{2}}{\rho w h} .
$$

With the dimensionless groups so formed, the relationship in Eq. 1 thus reduces to

$$
\omega / w=f(B i, P o, \text { geometric effects }) \text {. }
$$




\subsection{Significance of Dimensionless Groups}

The Biot number is a well-known dimensionless group encountered in the field of heat transfer. Its interpretation is the ratio of internal conduction resistance to heat transfer within a body to convection resistance external to the body. Thus, small Biot numbers represent negligible conduction resistance, supporting the validity of lumped element models in which entire bodies are assumed to have just one temperature at any point in time; that is, ignoring internal temperature gradients [13].

The thermo-electro parameter of Eq. 12 is more complex. The appearance of $V^{2} / \rho$ indicates a measure of electrical energy being converted to thermal energy via Joule heating, whereas the heat transfer coefficient appearing in the denominator represents the surroundings' corresponding ability to dissipate that heat. The combination $V^{2} / \rho w h$ has dimensions of temperature $(\theta)$, thus being a measure of the temperature increase of the actuator due to an applied voltage. Finally, multiplying by the thermal expansion coefficient $\alpha$ gives $P_{o}$, which is therefore also seen to be a measure of the overall thermal strain of the actuator.

\section{Discussion}

The dependence of tip deflection appears to be directly proportional to the thermo-electro coefficient, Po. We can see this by first considering the simplified model given in Adams and Layton [11]. According to this model, the tip deflection of the actuator in Fig. 2, is given by

$$
\frac{\omega}{w}=\frac{3 D \alpha H_{h} V^{2}}{h \rho H_{c}^{3}}\left[\frac{H_{h}}{\left(w+H_{h}\right)}\left(\frac{H_{c}}{H_{h}+H_{c}}\right)^{2}-\frac{H_{c}}{\left(w+H_{c}\right)}\left(\frac{H_{h}}{H_{h}+H_{c}}\right)^{2}\right]
$$

Recast in terms of $P o$,

$$
\frac{\omega}{w}=P o \frac{3 D H_{h} w}{H_{c}^{3}}\left[\frac{H_{h}}{\left(w+H_{h}\right)}\left(\frac{H_{c}}{H_{h}+H_{c}}\right)^{2}-\frac{H_{c}}{\left(w+H_{c}\right)}\left(\frac{H_{h}}{H_{h}+H_{c}}\right)^{2}\right],
$$

which clearly shows a liner dependence on Po.

The direct proportionality between $P o$ can be inferred from other works as well. Fig. 3 shows dimensionless tip deflection data from Huang and Lee [5] for a heat actuator of the type in Fig. 1. The near perfect curve fit demonstrates the linear dependence of deflection on the thermo-electro parameter. Indeed, the seminal paper of Comtois et al [1] also shows a linear dependence of deflection of $P o$ by virtue of deflection data being linearly dependent on dissipated electrical power.

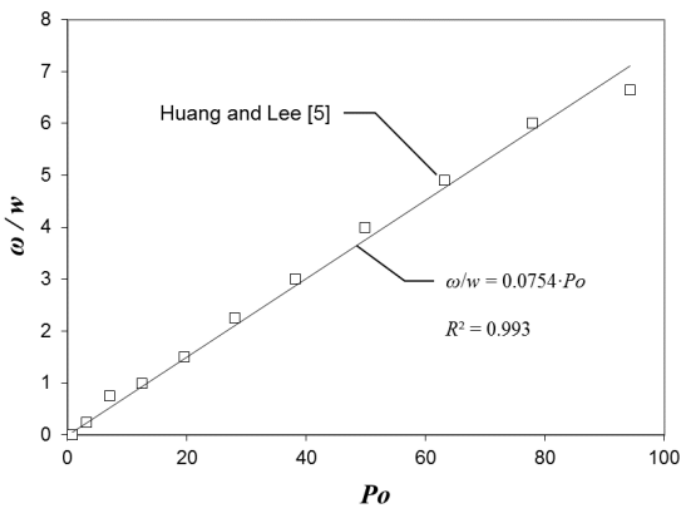

Fig. 3: Deflection data from Huang and Lee [5] shown in dimensionless form. The data are for an actuator of the type shown in Fig. 1 with a total length of $240 \mu \mathrm{m}$ and a flexure length of $60 \mu \mathrm{m}$. 
Also significant is the appearance of Biot number as a dependent dimensionless group. For the experimental parameters given in [5], typical values of Biot are calculated to be $B i \approx 0.001$. If an alternative length scale is used, say $L$ instead of $w$, these Biot numbers increase to $B i \approx 0.1-0.2$. Standard heat transfer references cite $B i<0.1$ as the typical condition for which a lumped element thermal model applies [13]. This implies that models in which detailed spatial variations of temperature are computed do not necessarily add extra predictive accuracy, at least for tip deflection. Indeed, Adams et al [11] well-predicts deflection while characterizing the actuator with only two temperatures, one for the hot arm and one for the cold arm/flexure. Naturally, predicting the locations of "hot spots" at which failures may occur due local extremes in temperature necessitates the use of more complex modelling strategies.

The most significant result of the preceding analysis, however, is the conspicuous absence of Young's modulus, $E$, in the resulting dimensionless groups. The dimensional analysis shows that the dimensions of $E$ cannot be accounted for, and therefore that the tip deflection cannot depend upon it. This proves the suspicions voiced in Adams and Layton [4] and Adams et al [11]. Nonetheless, the independence of actuator deflection and Young's modulus appears not to be generally recognized, as exemplified by virtually all analytical studies of hot arm actuation reporting the values of $E$ used in their respective models. (See [5]-[10], for example.) Most likely, the complexity of the models-usually consisting of several dozen simultaneous equations to be solved numerically - has obfuscated this fact.

\section{Conclusion}

A dimensional analysis of a generic MEMS hot arm actuator has been performed. The resulting dimensionless groups include a thermo-electro parameter, the Biot number, and a number of geometric groups. The thermo-electro parameter gages the relative importance of Joule heating to the energy dissipated to the surroundings, as well as the overall thermal strain of the actuator. Models of hot arm actuation and experimental data alike show tip deflection to be directly proportional the thermo-electro parameter. The Biot numbers encountered in hot arm actuators are on the order of 0.1 or less, bringing into question the need for detailed spatial temperature information in predicting deflection. Finally, the dimensional analysis proves that Young's modulus has no effect on actuator deflection, a fact that has gone unnoticed in the literature. Given these insights, new modelling schemes utilizing lumped element analysis and focusing on thermal deformation only may provide accurate results with less computational effort.

\section{References}

[1] J. H. Comtois, M. A. Michalicek, and C. C. Barron, "Electrothermal actuators fabricated in four-level planarized surface micromachined polycrystalline silicon," Sens. Actuators A, vol. 70, pp. 23-31, 1998.

[2] A. Siahmakoun, S. Kirkpatrick and Z. Roth, "Array of MEMS Mirrors for Beamforming," ASNE 2003 Symposium, Bloomington, IN, 2003.

[3] J. H. Comtois and V. M. Bright, "Applications for surface-micromachined polysilicon thermal actuators and arrays," Sens. Actuators A, vol. 58, pp. 19-25, 1997.

[4] T. M. Adams and R. A. Layton, Introductory MEMS: Fabrication and Applications. New York, NY: Springer, 2010.

[5] Q. A. Huang and N. K. S. Lee, "Analysis and design of polysilicon thermal flexure actuator," J. Micromech. Microeng., vol. 9, pp. 64-70, 1999.

[6] L. Li and D. Uttamchandani, "Modified asymmetric microelectrothermal actuator: analysis and experimentation," $J$. Micromech. Microeng. vol. 14, pp. 1734-1741, 2004.

[7] C. D. Lott, T. W. McLain, J. Harb, and L. Howell, "Thermal modeling of a surface-micromachined linear thermomechanical actuator," in Modeling and Simulation of Microsystems, pp. 370-373, 2001.

[8] R. Hickey, M. Kujath, and T. Hubbard, "Heat transfer analysis and optimization of two-beam microelectromechanical thermal actuators," J. Vac. Sci. Technol. A., vol. 20, pp. 971-4, 2002.

[9] N. D. Mankame and G. K. Ananthasuresh, "Comprehensive thermal modeling and characterization of an electrothermal-compliant microactuator," J. Micromech. Microeng., vol. 11, pp. 452-62, 2001.

[10] D. Yan, A. Khajepour and R. Mansour, "Modeling of two-hot-arm horizontal thermal actuator," J. Micromech. Microeng., vol. 13, pp. 312-322, 2003.

[11] T. M. Adams, A. J. Bomar, A. J., and S. Kirkpatrick, "Lumped Element Model for a MEMS Hot Arm Actuator," in Proceedings of the CSME Forum, Canada, 2010.

[12] B. R. Munson, D. F. Young, T. H. Okiishi, Fundamentals of Fluid Mechanics, 8th Ed. Hoboken, NJ: J. Wiley \& Sons, 2016. 
[13] Y. Çengel and A. J. Ghajar, Heat and Mass Transfer: Fundamentals and Applications, 5th Ed. New York, NY: McGraw Hill, 2014. 